\section{Information Storage Using Supramolecular Surface Patterns}

Novel strategies for information storage technology rely upon multistable systems that can be controllably switched between different configurations of comparable free energy. Multistability is present in many molecular and supramo(conformations, redox and spin states, shape and dimensionality) that can be influenced by external stimuli. In the solid state, however, it is difficult to amplify the effects of molecular switching to higher length scales in order to relay the response to the outside world.

Rotaxanes (1) are molecules in which a macrocycle is mechanically locked onto a "thread" by two bulky "stoppers." Their architecture, analogous to that of an abacus, suggests that they could be used as switchable components for artificial machines (2) that function through mechanical motion at the molecular level $(3,4)$. Controlling such motion in the solid state could be used to store information.

Thin films (3- to 35-nm thickness) of each of three amide-based rotaxanes (1 to $\mathbf{3}$, Scheme 1) (5) were grown by drop casting and postthermal annealing onto graphite and mica. The films can be imaged by atomic force microscopy (AFM) in contact mode with a set point force below a threshold value of $2 \mathrm{nN}$. They exhibit a homogeneous morphology over $\mathrm{a} \mathrm{cm}^{2}$ area and are stable over a period of several months.

An increase in the load force to just above the 2-nN threshold results in a mechanical perturbation whose effect is localized at the contact area of the tip. When the tip is continuously scanned along a line, a string of regularly spaced dots appears. The dots emerge upon repeating the line scan a number of times (between 4 and 20) depend-

Scheme 1.

\section{Massimiliano Cavallini, ${ }^{1}$ Fabio Biscarini, ${ }^{1}$ Salvador León, ${ }^{2}$ Francesco Zerbetto, ${ }^{2}$ Giovanni Bottari, ${ }^{3}$ David A. Leigh ${ }^{3}$} lecular systems through a variety of properties

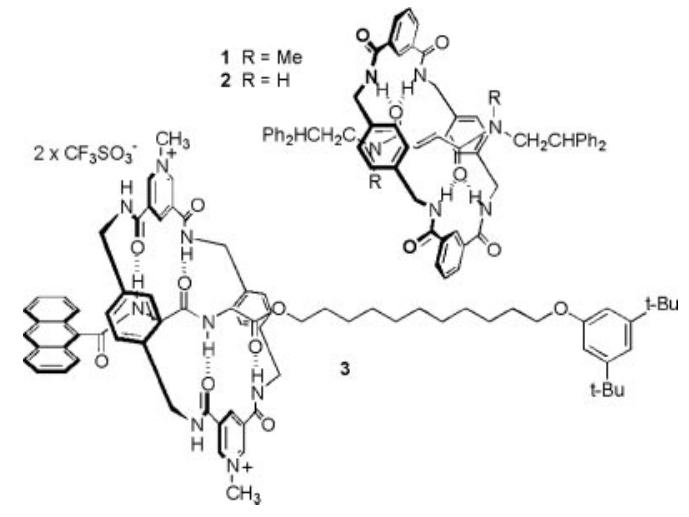

ing on the scan rate (typically 1 to $2 \mathrm{~Hz}$ ). Scanning a series of lines results in a regular array of dots of uniform width, height, and pitch (Fig. 1A). The number of dots is proportional to the length of each line scan (Fig. 1B), so that any predetermined number of dots can be reliably fabricated on the surface. The film thickness controls the dot size and the spacing and hence the density of dots per unit line; the thinner the film, the denser and smaller the dots are. The transformation can be induced over a large area (Fig. 1C), and it produces robust features even in the presence of surface steps and terraces. The linear dependence on the scan length allows one to write information as strings of bits (Fig. 1D).

The effect of the mechanical perturbation on the film appears during line scans as a roughening of the topographical profile, with the position of the dots fixed at early stages. The transformation can be interrupted and restarted by turning off and on the perturbation. No sign of scraping or wear of the film is observed, ruling out plowing with the AFM tip, which instead occurs as the set point force exceeds 3 to $4 \mathrm{nN}$.

The transformation takes place only with the rotaxanes, not the component threads or macrocycles

alone, and is thus intrinsically related to the mechanically interlocked architecture. Molecular modeling of the solid state rotaxane structure (4) shows the existence of two nearly degenerate surfaces, (100) and (101), which can be switched with a relatively low activation energy $\left(200.4 \mathrm{~kJ} \mathrm{~mol}^{-1}\right)$ by circumrotation of the macrocycles. The collective fabrication of dots arises from coupled nucleation recrystallization favored by the ease of intercomponent mobility in the solid state. The scanning AFM tip gives the energy to the molecules along the line to reorganize into nuclei. As nuclei coarsen by ripening, a characteristic distance emerges. Finally, stable nuclei grow by incorporation of the mobile molecules to form the dots.

Information storage by writing individual dots with a scanning probe has previously been demonstrated $(6,7)$. Our approach enables the writing of multiple dots simultaneously. This could make it suitable for scaling up to a lithography based on multiple sources of perturbation, for instance by using a stamp. With such an approach, information storage on a thin film could reach densities of 10 to $100 \mathrm{Gbit} / \mathrm{in}^{2}$
Fig. 1. (A) Array of dots fabricated of 1 by individual line scans of the AFM tip on a 5-nm-thick film of 1 deposited on highly oriented pyrophite. (B) For a given thickness (here $20 \mathrm{~nm}$ ), the the scan length. The Film thickness controls the characteristic size. Varying the film thickness in the range between 3 and $35 \mathrm{~nm}$, interdot distance increases from 100 to $500 \mathrm{~nm}$, the dot full-width-half-maximum concept for information storage. The sequence "e c 7 a 8 " in the hexadecimal base corresponds to the number 968616 .

References and Notes

1. J.-P. Sauvage, C. Dietrich-Buchecker, Eds. Molecular Catenanes, Rotaxanes and Knots (Wiley-VCH, Weiheim, Germany, 1999).

2. Molecular Machines Special Issue, J. F. Stoddart ed., Acc. Chem. Res. 34, 410 (2001).

3. C. P. Collier et al., Science 285, 391 (1999)

4. F. Biscarini et al., J. Am. Chem. Soc. 124, 225 (2002).

5. F. G. Gatti et al., J. Am. Chem. Soc. 123, 5983 (2001).

6. R. García et al., J. Appl. Phys. 86, 1898 (1999).

7. G. Binnig et al., Appl. Phys. Lett. 74, 1329 (1999).

${ }^{1}$ Consiglio Nazionale delle Ricerche-ISMN, Sezione di Bologna, Via P. Gobetti 101, I-40129 Bologna, Italy. 2Dipartimento di Chimica "G. Ciamician," Universitá degli Studi di Bologna, Via F. Selmi 2, I-40126 Bologna, Italy. ${ }^{3}$ School of Chemistry, University of Edinburgh, The King's Buildings, West Mains Road, Edinburgh EH9 3JJ UK. 